\title{
Contribution of industrial composite parts to fatigue behaviour simulation
}

\author{
Ara Sedrakian ${ }^{a}$, Tarak Ben Zineb ${ }^{\text {b,*, }}$, Jean Louis Billoet ${ }^{\text {a }}$ \\ a LM2S-UPRESA CNRS 8007, ENSAM Paris, 151 Boulevard de l'Hôpital, 75013 Paris, France \\ ${ }^{\mathrm{b}}$ LPMM-UMR CNRS 7554, ENSAM Metz, 4 rue Augustin Fresnel, 57078 Metz Cedex 3, France
}

\begin{abstract}
The dimensioning of composite structures presents the problem of behaviour modelling. This problem comes not only from the specific nature of the involved materials, but also from the reproducibility of the process both on the series scale as well as on the specimen characterisation level, which enables determination of the modelling parameters. In the case of static dimensioning with or without damage, the reproducibility of the mechanical parameters is satisfactory overall, regardless of the implemented experimental procedure. In fatigue dimensioning, the two aspects; process influence and procedure of identification play a major role in the application of the lifetime predictive models. This research has, as a medium-term objective, to partially answer the problems raised previously. The damage model used is based on generalised standard material thermodynamics. This model, coupled with the finite element method, allows simulation of the fatigue behaviour of composite parts.
\end{abstract}

Keywords: Fatigue; Damage model; Iterative approach; Identification; Experimental tests; Finite element method

\section{Introduction}

Nowadays more and more composite parts subjected to fatigue loading (spring leaf of commercial vehicles, engine components and plane wing elements etc.) are to be found in operation. When designing these structures, high development costs arise. In the absence of a reliable model of lifetime prediction, evaluation of the fatigue behaviour is always carried out on real parts. This naturally implies:

- Design and mould manufacture for the production of composite parts.

- Composite part production process.

- The design of specific test benches dedicated to the study of fatigue behaviour.

- The tests take a long time (on average 2 months of tests for a fatigue curve).

It will consequently be advisable to provide designers with dimensioning rules and models of lifetime predic-

* Corresponding author. Tel: +33-3-8737-5434; fax: +33-3-87375470.

E-mail address: tarak.benzineb@metz.ensam.fr (T. Ben Zineb). tion guaranteeing a maximum level of reliability. Accordingly, earlier investigations showed that lifetime prediction methods generally used in the case of homogeneous materials, like the Dang Van [1,2] criterion, are ineffective for composite materials. Mechanical degradation occurs without a significant loss of rigidity. Transverse cracking is one of the causes. It is often this phenomenon which first appears in a diffuse way and which, without being really penalising, entails the development of other damage, like the delamination and rupture of fibres. Those are more detrimental to the mechanical strength of the composite. Initiation and propagation of micro-cracks primarily depend on associated parameters:

- manufacturing process

- nature of the basic components

- loading mode.

For composite structures presenting high stress concentrations primarily due to large thickness gradients, the application of complex loading, etc., material damage is often accompanied by stress redistribution phenomena. These may cause a rapid material degradation. Consequently, in modelling damage evolution this phenomenon should be taken into account. Indeed, the initiation 
and propagation of the damage related to high stress concentrations have a significant influence on strain and stress states during the lifetime and thus on the final endurance limit. The quantification of the latter, while being based only on the initial stress field, can be dangerous since the result turns out to be very approximate $[3,4]$. Consequently, in fatigue modelling, a damage model with updated strain and stress fields appears to be essential. The choice of a representative model thus constitutes an important point of this study. The characterisation and identification of the predictive model parameter imply a choice of fatigue tests and suitable identification procedures. As an example, during the characterisation of the fatigue behaviour of a composite glass/epoxy in the fibre direction, the structure effect is hardly avoided.

The aim is to develop an approach based on a representative damage model. This model must be able to take into account all material, test and environment parameters, data scattering and especially the stress redistribution phenomenon. Since the seventies, great quality works have been undertaken seeking these models. In this field, there are two modelling approaches:

- models based on the resistance loss notion [5-7], and using quadratic criteria $[8,4]$, for the determination of lifetime. These models showed insufficiencies when taking into account damage mechanisms associated with a multiaxial stress and strain field.

- models using the generalised standard material thermodynamics to define damage evolution laws [9-11]. The representativeness of these models is related to the judicious choice of the modelling scale.

The major difficulty of these two approaches lies in the definition of characterisation tests and identification procedures. For several years, research has been rather directed towards a clearer identification of degradation mechanisms aiming at better modelling.

\section{Context of modelling and bases}

\subsection{General information on the approach suggested}

The material is a composite containing unidirectional glass fibres and an epoxy matrix. The manufacturing process is resin transfer moulding (RTM). Applications, made by this composite, are subjected to sinusoidal loading. The loading ratio $R=\operatorname{Load}_{\text {min }} / \operatorname{Load}_{\text {max }}$ is between 0 and 1 . In the following we only consider undulating fatigue loading.

With regard to the parameters influencing the fatigue behaviour of the material studied, only the loading level will be taken into account [12]. Thus three behaviours resulting from the fatigue tests will be distinguished:
Low cycle fatigue, the limited and unlimited endurance (Fig. 1). The parameters, loading ratio $R$, frequency $f$, temperature $T$ and humidity, are fixed [13-15].

\subsection{Material scale with damage gradient on the ply}

The damage in the fatigue of the composites is characterised by a progressive rigidity. The rigidity loss corresponds to the behaviour degradation. Consequently, when modelling the fatigue phenomenon a damage model coupled to a structural analysis is essential. The use of the damage mechanics requires the introduction of generalised standard materials thermodynamics on the one hand and the definition of a material modelling scale on the other hand. To use classical mechanical approaches, an equivalent homogeneous behaviour will be preferred. The composite will be considered as a periodic structure and averages will be carried out over one elementary period, considered as statistically representative of the heterogeneous medium. Unfortunately, this does not entirely solve the problem for two reasons:

- It is difficult to have full knowledge of the component characteristics (fibre, matrix).

- The interactions of the matrix-fibre interface are not well represented.

To make up for these shortcomings, new approaches represent as accurately as possible the physical phenomena which find their origin in the material heterogeneity. These approaches establish the link between pilot mechanisms on a microscopic scale and the physical sizes translating the behaviour of the structure on a macroscopic scale. To that purpose, the approach developed by Ladevèze [16], based on the theory of anisotropic damage, attempts to describe the damage mechanisms occurring in the behaviour on a mesoscopic scale which is that of the elementary ply. In this meso-modelling it should be noted that:

- such a model is semi-discrete and thus differs from the conventional continuum mechanics models.

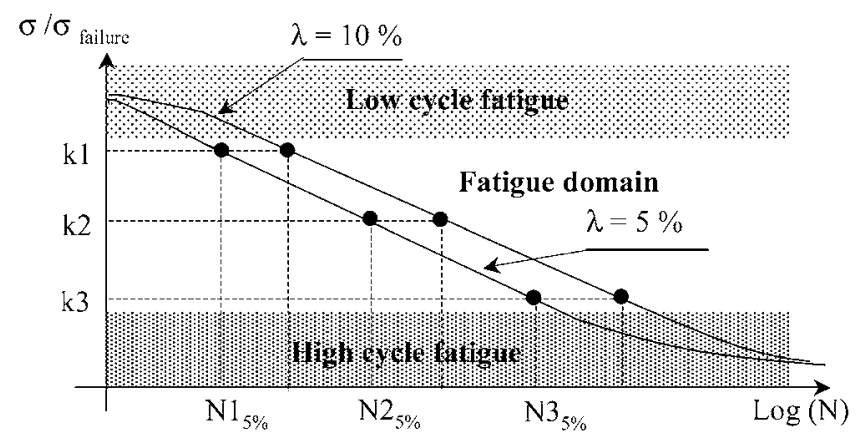

Fig. 1. Wöhler curves $\left(N i_{\lambda \%}\right.$ is the number of cycles for a $\lambda \%$ rigidity loss at $k i$ loading level) 
- these models are not invariant by scaling change.

- the damage state on a mesoscopic scale is locally imposed evenly on each meso-component.

However the micro-cracking of the matrix and of the matrix-fibre interfaces are not necessarily uniform in the ply thickness. For example during a four or three point bending test, the stress and strain state is characterised by a gradient on the elementary ply, consequently the resulting damage cannot remain uniform on each ply. It is then difficult to define the size of the elementary volume through a homogeneous state, which is to be solved. Such an analysis is related to the concept of continuum mechanics. An easy behaviour identification and a true homogeneity of the stress and strain state within this element must justify the definition of this volume element. The use of the finite element method can partially overcome this obstacle. The behaviour of each finite element at each loading cycle " $N$ " is characterised by the initial behaviour of the elementary ply associated to a localisation function:

$C_{i j k l}^{\mathrm{el} k}(N)=C_{\text {meso }}^{0} f($ localisation, $N)$

\subsection{Analysis of the stress and strain field updating}

The orientation and provision of fibres of a unidirectional ply and its loading direction can be at the origin of a triaxial stress state. Taking into account this multiaxiality in the modelling of the fatigue behaviour constitutes most of the basic reflections. Besides this multiaxiality poses the problem of the uniformity of the strain and stress field. As an example, the initiation and the propagation direction of the damage cannot be the same for a unidirectional ply with $\theta$ degrees and the same one with a central hole, both loaded in tension. In this last case, taking the redistribution stress phenomenon and load transfers into account is as significant as choosing the predictive model. The work of Shokrieh et al. [4] showed that in the case of a unidirectional fold with $\theta^{\circ}$ loaded in tension, the strain remains homogeneous within the sample. A polynomial criterion of fatigue without updating of the strain field is amply sufficient for the lifetime prediction.

However, a thorough study undertaken by Asundi and Chow [3], highlighted the variation of the stress and strain state according to the damage during a static tensile test on a laminate having a central hole. The Moiré interferometric method was used to quantify the effect of the damage on the redistribution and the variation of the strain around the hole (Fig. 2). Although this phenomenon was demonstrated in the static loading case, it will be assumed to occur in the fatigue loading case. Consequently for the modelling of fatigue behaviour, the predictive model must be based on an analysis cycle by

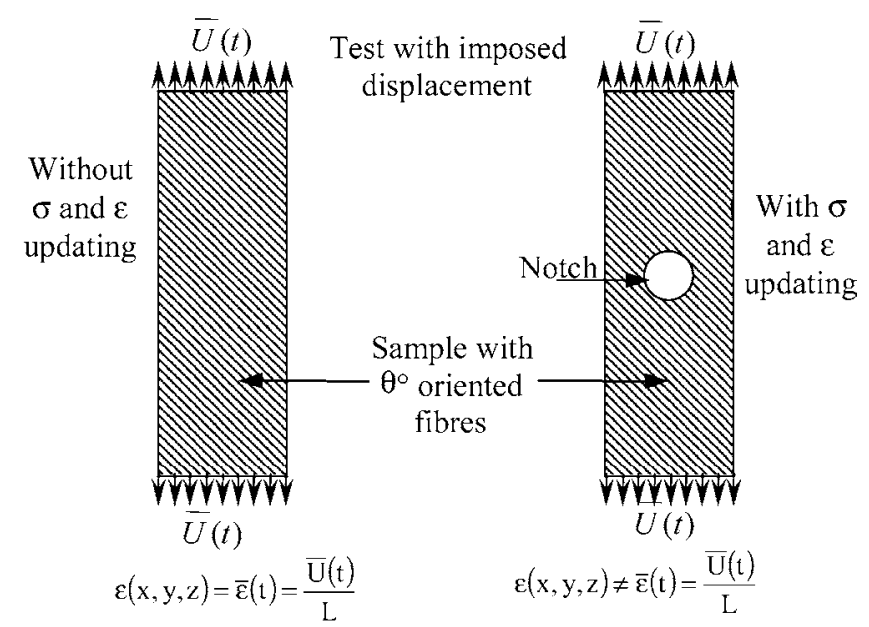

Fig. 2. Approach with or without strain redistribution.

cycle or if required, per package of cycles in order to reduce the computing time.

\section{Three dimensional damage model}

The damage model used was developed by Ladevèze [10], and exploited by Thionnet [11] and Linhonne [17], inter alia. It is based on a homogenised elementary mesoscopic vision of the damage state. This model is used in the fatigue case even it is primarily established in the static case. Nevertheless, according to Reifsnider [7] and Guilmineau [18], it is possible to suppose the physical and geometrical similarity of defects in static and fatigue cases. In order to make the numerical implementation in FE software easier, the model is formulated in strains [17]. The damage evolution is formulated in an explicit way. The model respects the symmetry of the rigidity matrix associated with the incremental form of the constitutive laws.

\subsection{General context}

The model presented is developed within the framework of the thermodynamics of the irreversible phenomena, and under the assumption of the thin or moderately thick structures $\left(\sigma_{33}=0\right)$, it is formulated in strains. Thermal phenomena are neglected. The model is formulated with the assumption of small perturbations but can be applied to non-linear geometrical formulations. The volumic energy of elastic strain, which is taken as a thermodynamics potential, is given by:

$$
\begin{aligned}
2 W_{\mathrm{e}} & =C_{11} \varepsilon_{11}^{2}+2 C_{22} v_{12} \varepsilon_{11} \varepsilon_{22}+C_{22} \varepsilon_{22}^{2}+G_{12} \gamma_{12}^{2} \\
& +G_{13} \gamma_{13}^{2}+G_{23} \gamma_{23}^{2}
\end{aligned}
$$

where $C_{i j}$ and $G_{i j}$ represent the elastic behaviour constants, and with: 


$$
\begin{aligned}
C_{11} & =\frac{E_{1}}{1-v_{12} v_{21}} C_{22}=\frac{E_{2}}{1-v_{12} v_{21}} \gamma_{12}=2 \varepsilon_{12} \gamma_{13}=2 \varepsilon_{13} \gamma_{23} \\
& =2 \varepsilon_{23}
\end{aligned}
$$

The symmetry relations arising from the existence of a thermodynamic potential give:

$\frac{C_{11}}{C_{22}}=\frac{E_{1}}{E_{2}}=\frac{v_{12}}{v_{21}}$ it comes $v_{21} C_{11}=v_{12} C_{22}$

and according to the definition of $C_{11}$ one notices that:

$$
\begin{aligned}
& C_{11}\left(1-v_{12} v_{21}\right)=E_{1} \Rightarrow C_{11}=E_{1}+v_{12} v_{21} C_{11}=E_{1} \\
& \quad+v_{12}^{2} C_{22}
\end{aligned}
$$

In this form, the value of the stress $\sigma_{22}=C_{22}\left(\varepsilon_{22}+v_{12} \varepsilon_{11}\right)$ appears clearly. The elastic deformation energy (2) can thus be written as follows:

$$
\begin{aligned}
2 W_{\mathrm{e}} & =E_{1} \varepsilon_{11}^{2}+C_{22} v_{12}^{2} \varepsilon_{11}^{2}+2 v_{12} C_{22} \varepsilon_{11} \varepsilon_{22}+C_{22} \varepsilon_{22}^{2} \\
& +G_{12} \gamma_{12}^{2}+G_{13} \gamma_{13}^{2}+G_{23} \gamma_{23}^{2} \\
& =E_{1} \varepsilon_{11}^{2}+C_{22}\left(\varepsilon_{22}+v_{12} \varepsilon_{11}\right)^{2}+G_{12} \gamma_{12}^{2} \\
& +G_{13} \gamma_{13}^{2}+G_{23} \gamma_{23}^{2}
\end{aligned}
$$

\subsection{Damage variables}

One seeks to build a model on the mesoscopic level based on the degradation characteristics at the micro level. The physical observations of the damage modes of the materials used enable us to define the damage variables by adopting the following assumptions:

- The damage in the glass/epoxy composites occurs according to three modes:

fibre failure

transverse and longitudinal cracking of the matrix rupture on the fibre/matrix interface

- The initiation and the propagation of delamination will not be considered in this model.

- In transverse compression the cracks tend to close up again and do not create any additional damage. In the fibre direction, the damage due to the localised buckling of fibres loaded in compression is neglected.

- For lack of experimental information on the shear moduli in 13 and 23 planes, they considered undergoing an evolution similar to the modulus in the 12 plane. The $C_{22}$ and $E_{1}$ moduli will be affected respectively by the variables $d_{22}$ and $d_{11}$.

The elastic moduli of the material are [17]:

$$
\begin{array}{ll}
E_{1}=E_{1}^{0}\left(1-d_{11}\right) ; & C_{22}=C_{22}^{0}\left(1-d_{22}\right) \\
G_{12}=G_{12}^{0}\left(1-d_{12}\right) ; & G_{13}=G_{13}^{0}\left(1-d_{13}\right) ; \quad G_{23}=G_{23}^{0}\left(1-d_{23}\right)
\end{array}
$$

where the superscript 0 indicates the virgin state of the material.

\subsection{Evolution laws}

The introduction of damage variables into expression (5) gives Eq. (7) which represents the damaged deformation energy. This equation will be used throughout the study.

$$
\begin{aligned}
& 2 W_{\mathrm{e}}=E_{11}^{0}\left(1-d_{11}\right) \varepsilon_{11}^{2}+C_{22}^{0}\left\langle\varepsilon_{22}+v_{12} \varepsilon_{11}\right\rangle_{-}^{2}+C_{22}^{0}(1 \\
& \left.-d_{22}\right)\left\langle\varepsilon_{22}+v_{12} \varepsilon_{11}\right\rangle_{+}^{2}+G_{12}^{0}\left(1-d_{12}\right) \gamma_{12}^{2}+G_{13}^{0}(1 \\
& \left.-d_{13}\right) \gamma_{13}^{2}+G_{23}^{0}\left(1-d_{23}\right) \gamma_{23}^{2}
\end{aligned}
$$

Transverse matrix cracks are supposed to be fully closed again during compression loading and do not have any influence on the damage evolution. But during tension loading they contribute to the damage evolution. Consequently, expression (7) has two distinct parts $(\langle\rangle+)$ and $(\langle\rangle-)$ respectively representing the positive and negative parts of the expression. The evolution laws of the damage variables are given by introducing the thermodynamics of generalised standard materials. The thermodynamic variables associated to the damage variables $d_{i j}$ are thus defined by:

$Y_{i j}=-\frac{\partial W_{\mathrm{e}}}{\partial d_{i j}}$

The evolution of damage in composite materials can be summarised in three stages. In the first stage rapid stiffness changes occur until the stiffness reaches a plateau. In the second stage, this evolution is very slow. It is due to the appearance of microscopic cracks, which start propagate. In the third stage, the density of microscopic cracks becomes significant. Macro-cracks appear and the damage increases rapidly.

To approach this evolution numerically, several laws are proposed $[19,20]$, etc. They are of power type and generally use the thermodynamic variables associated with the internal variables to express damage evolution. These laws present a more or less significant number of parameters. The major difficulty lies in the experimental identification, and in the physical interpretation of these parameters. For this study, Norton's evolution law is used (9). The latter comprises two parameters associated with each damage variable, that is to say ten on the whole.

$$
\begin{aligned}
\varphi= & \frac{\alpha_{11}}{1+\beta_{11}} Y_{11}^{\beta}{ }_{1}+\frac{\alpha_{22}}{1+\beta_{22}} Y_{22^{2}}^{\beta}+\frac{\alpha_{12}}{1+\beta_{12}} Y_{12}^{\beta}+\frac{\alpha_{13}}{1+\beta_{13}} Y_{13}^{\beta_{13}} \\
& +\frac{\alpha_{23}}{1+\beta_{23}} Y_{23^{3}}^{\beta_{3}}
\end{aligned}
$$


The major interest of this potential lies in the fact that it is of class $C^{\infty}$. The singular problems encountered in the case of potentials with threshold, used generally for the modelling of static damage, are avoided. The parameters $\left(\alpha_{i j} ; \beta_{i j}\right)$ of dissipation potential are influenced by the loading conditions $[14,13,12]$, i.e. by the ratio $R$ and the temperature, as well as by intrinsic material properties. Nevertheless, in this investigation the effect of temperature is neglected.

Damage rates result from the potential of dissipation by using the following relations.

$$
\begin{aligned}
& \frac{\mathrm{d}\left(d_{11}\right)}{\mathrm{d}(N)}=\frac{\partial \varphi}{\partial Y_{11}}=\frac{\alpha_{11} \beta_{11}}{1+\beta_{11}}\left(\frac{1}{2} E_{1}^{0} \varepsilon_{11}^{2}\right)^{\beta_{11}-1} \\
& \frac{\mathrm{d}\left(d_{22}\right)}{\mathrm{d}(N)}=\frac{\partial \varphi}{\partial Y_{22}}=\frac{\alpha_{22} \beta_{22}}{1+\beta_{22}}\left(\frac{1}{2} C_{22}^{0}\left(v_{12} \varepsilon_{11}+\varepsilon_{22}\right)^{2}\right)^{\beta_{22}-1} \\
& \frac{\mathrm{d}\left(d_{12}\right)}{\mathrm{d}(N)}=\frac{\partial \varphi}{\partial Y_{12}}=\frac{\alpha_{12} \beta_{12}}{1+\beta_{12}}\left(2 G_{12}^{0} \varepsilon_{12}^{2}\right)^{\beta_{12}-1} \\
& \frac{\mathrm{d}\left(d_{13}\right)}{\mathrm{d}(N)}=\frac{\partial \varphi}{\partial Y_{13}}=\frac{\alpha_{13} \beta_{13}}{1+\beta_{13}}\left(2 G_{13}^{0} \varepsilon_{13}^{2}\right)^{\beta_{13}-1} \\
& \frac{\mathrm{d}\left(d_{23}\right)}{\mathrm{d}(N)}=\frac{\partial \varphi}{\partial Y_{23}}=\frac{\alpha_{23} \beta_{23}}{1+\beta_{23}}\left(2 G_{23}^{0} \varepsilon_{23}^{2}\right)^{\beta_{23}-1}
\end{aligned}
$$

in which $N$ represents the number of cycles at a given time.

\subsection{Computation of the strain and stress fields}

In the case of a simple geometry and boundary conditions, the calculation of the stress field can be done analytically. This is not valid for an industrial case. For the latter, a computation by the finite element method of all $\Delta N$ numerical cycles is necessary in order to have the most realistic macroscopic stress field in the structure. The variation in the number of cycles $\Delta N$ depends on the damage rate of the structure, Eqs. (10) to (14). The numerical loading level represents the maximum level of the experimental loading diagram (Fig. 3). The elastic stresses computed by FEM can be updated at each iteration.

Strain and stress fields can in part present gradients requiring a mesh that is sufficiently refined. The influence of the loading ratio $R$ is taken into account in the evolution laws of the damage model. This approach is only applicable in the case of fatigue tests characterised by a stress ratio $R$ ranging between 0 and 1 (undulated fatigue). Indeed, damage mechanisms are not identical during a tension-tension fatigue test and a tension-compression one. The simulation of alternate tests $(R<0)$ would require taking this difference into account. During experiments, fatigue tests on structures are stopped according to a loss criterion on total rigidity. This criterion is fixed by a condition specification and generally ranges between 5 and $10 \%$ of rigidity loss. The test is monitored in displacement. The global induced force is measured and a rigidity loss is computed. With regard to the approach presented here, iterations stop as soon as the criterion of $10 \%$ of rigidity loss is reached numerically.

\section{Identification of the damage model parameters}

\subsection{Experimental methods: test selection}

Damage model identification strongly depends on the modelling scale. The identification of a mesoscopic model does not require the same experimental procedures as a microscopic one. The complex characterisation of micro-modelling lies in the knowledge of the component characteristics which requires very expensive experimental means. In contrast, the identification of the mesoscopic models is based on a reasonable scale. It is the elementary ply whose equivalent homogeneous behaviour is identifiable by simple experimental means. This identification is valid provided that the test used is representative of the elementary volume defined while modelling, i.e. characterised by homogeneous strain and stress fields. Variables " $d_{i j}$ " of the damage model are directly related to the modulus of homogenised material. Three series of fatigue undulated tests characterised by specific damage modes must be defined:

- Tests characterised by a fall of the homogeneous modulus $E_{1}$ (ruptures of fibres and fibre/matrix interfaces)

- Tests influencing the $C_{22}$ modulus (damage of the matrix)

- Plane or interlaminar shear tests influencing a decrease of the $G_{12}, G_{13}$ or $G_{23}$ moduli.

Hahn [21], Lee S. [22] and Boehler [23] propose various types of tests to study the composite behaviour under shear loading (both inter- and intra-laminar). These tests have complex modes of loading (torsion, biaxial tension-compression) or quite simply, the loading in monotonous tension, by exploiting the anisotropic nature of the material. Among the tests usually used for the characterisation of unidirectional composite behaviour, we notice:

- tensile tests on samples fitted with aluminium tabs at each end (oblique or right tabs) [4]

- three point bending test with distant supports

- four point bending tests.

Acute attention must be given to the characterisation of the fatigue behaviour in the fibre direction. The identification of this behaviour must leave aside all phenomena 


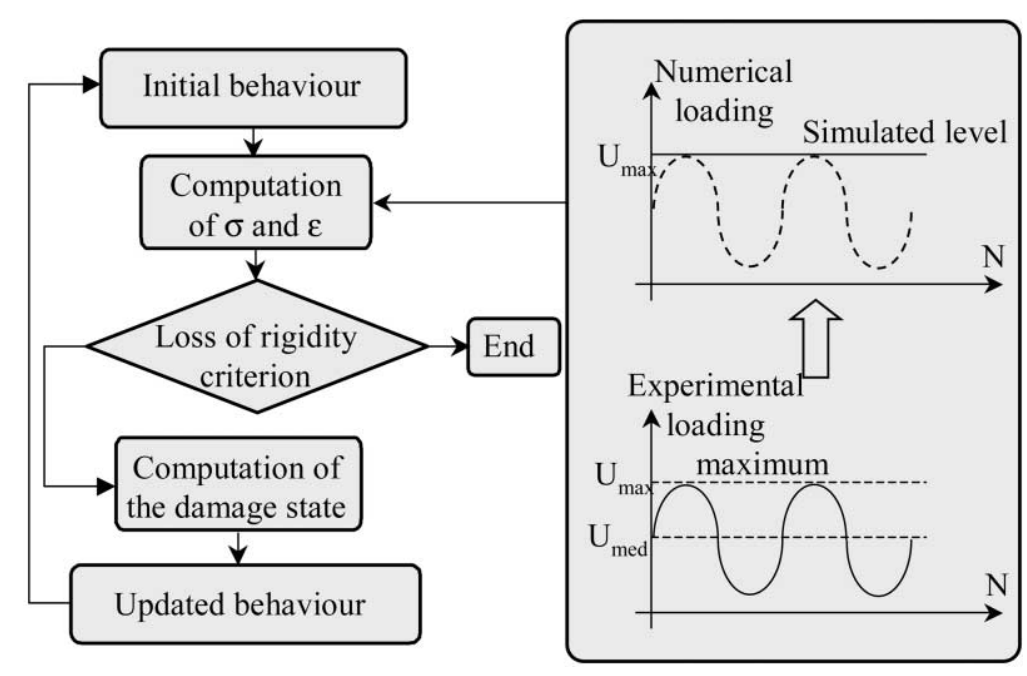

Fig. 3. Approach with strain and stress updating. The loading applied numerically is constant and corresponds to the maximal value of the experimental loading.

of "material-structure" coupling. Former studies [24] showed the existence of a "structure effect" during characterisation of the composite in the fibre direction by using 3- or 4-point bending tests. Indeed, the nonhomogeneous character of the strain and stress fields of the latter generates a low sensitivity to the damage and the resulting behaviour is not representative. Conversely, the homogeneous character of the strain and stress fields for a tensile test makes it possible to regard them as being more representative of the studied phenomenon, but also more sensitive to micro-defects. The representativeness of the tensile tests is closely related to the quality of the samples used. Consequently, the choice of tests for the characterisation of the fatigue behaviour in the fibre direction becomes difficult. Tensile tests are used to identify the damage in spite of the sensitivity to micro-defects.

In the same way, classification tests of shear behaviour must present a homogeneous character. Accordingly, we notice that the laminate $[ \pm 45]_{\mathrm{eq}}$ (the index eq. means equilibrate) loaded in uniaxial tension is a case of loading in quasi-pure shear of the elementary ply. It has a transverse yield stress in tension or compression higher than the shear yield stress. It may be expected to be damaged only according to the shear stress. Finally, a $90^{\circ}$ unidirectional tension is also a case of homogeneous loading and allows identification of the fatigue behaviour in the transverse direction. These tests can also be very sensitive to the micro-defects existing in the matrix and in the fibre/matrix interface.

\subsection{Identification procedure}

\subsection{1. "Modified Basquin" diagram}

To identify the $\alpha_{i j}$ and $\beta_{i j}$ coefficients, the high cycle behaviour (for $10^{3}<N<10^{6}$ ) of the glass/epoxy material in the fibre direction, transverse direction and shear is used. In order to take into account the scattering of the experimental results on the one hand and to facilitate the identification on the other hand, the "modified Basquin" model is used. This model is based on a logarithmic diagram and expresses the evolution of the strain vs lifetime. One modified Basquin diagram is necessary for each of the three fatigue behaviours: longitudinal, transverse direction and shear. All fatigue tests are monitored in displacement. For each loading level 3-5 samples are tested according to the scattering of the results. In the modified Basquin diagram, the imposed maximum strain vs lifetime is carried out for each behaviour studied (Fig. 4):

$$
\ln \varepsilon_{i j \max }=a_{i j}+b_{i j} \ln N_{x \%} \quad(i=1,2 j=1,2)
$$

$N_{x \%}$ accounts for the lifetime at 5 or $10 \%$ of rigidity loss. The $a_{i j}$ and $b_{i j}$ coefficients represent the slope and ordinate at the origin of the modified Basquin line, respectively.

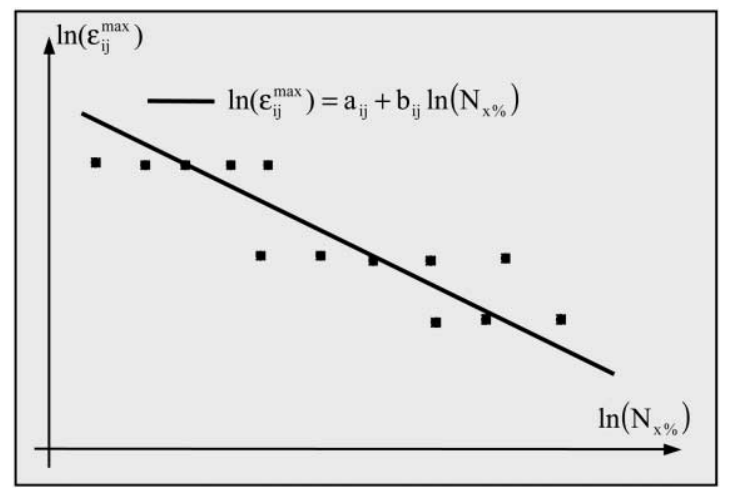

Fig. 4. Modified Basquin diagram. 


\subsubsection{Example: identification of $\alpha_{11}$ and $\beta_{11}$}

The first stage of the identification consists in determining the modified Basquin line equation. In the fibre direction the modified Basquin diagram is written:

$\ln \varepsilon_{11 \max }=a_{11}+b_{11} \ln N_{x \%}$

For a tensile test in fatigue according to the longitudinal direction, the damage rate equation is written (Eq. (10)):

$\frac{\partial d_{11}}{\partial N}=\frac{\alpha_{11} \beta_{11}}{1+\beta_{11}}\left(\frac{E_{1}^{0}}{2} \varepsilon_{11}^{2}(N)\right)^{\beta_{11}-1}$

In this case, the loading is homogeneous, the displacement is imposed, and $\varepsilon_{11}$ is constant. Integration of the previous equation gives:

$d_{11}=N \frac{\alpha_{11} \beta_{11}}{1+\beta_{11}}\left(\frac{E_{1}^{0}}{2} \varepsilon_{11}^{2}\right)^{\beta_{11}-1}$

The identification can be carried out on modified Basquin lines corresponding to 5 or $10 \%$ of rigidity loss. In this precise case, $d_{11}=0.1$ (to facilitate the writing we consider $d_{11}=D$ ) and $N=N_{10 \%}$. Eq. (18) gives:

$D=k_{1}\left(\frac{E_{1}^{0}}{2} \varepsilon_{11}^{2}\right)^{k_{2}} N$ where $k_{1}=\frac{\alpha_{11} \beta_{11}}{1+\beta_{11}}$ and $k_{2}=\beta_{11}$

$-1$

Taking the Napierian logarithm of Eq. (19) leads us to:

$\ln \left(\varepsilon_{11 \max }\right)=\frac{1}{2 k_{2}} \ln \left[D / k_{1}\left[\frac{E_{1}^{0}}{2}\right]^{k_{2}}\right]-\frac{1}{2 k_{2}} \ln (N)$

By comparing Eqs. (16) and (20) we finally write:

$\beta_{11}=\frac{-1}{2 b_{11}}+1$ and $\alpha_{11}=\frac{\left(1+\beta_{11}\right) D}{\beta_{11} e^{2\left(\beta_{11}-1\right) a_{11}}}\left[\frac{2}{E_{1}^{0}}\right]^{\beta_{11^{-1}}}$

Coefficients $\alpha_{11}$ and $\beta_{11}$ are identified by using relation (21). Fig. 5 presents in a synthetic way all the stages allowing the complete parameter identification of the various evolution laws.

\subsection{Characterisation tests}

For usual devices of fatigue by tension, the tabs usually used on the sample are at the origin of premature failures by shear stress. They occur at the level of the junction between the tabs and the sample [24]. RENAULT has developed a test device in collaboration with ONERA. It allows the fixing of the sample in grips by a system exerting a non-uniform pressure in order to avoid fibre wrenching in the useful part of the specimen. The recourse to tabs could thus be avoided. All the characterisation tests in static and fatigue cases were carried out by the "Université Technologique de Compiègne" and RENAULT. In this paper no description of

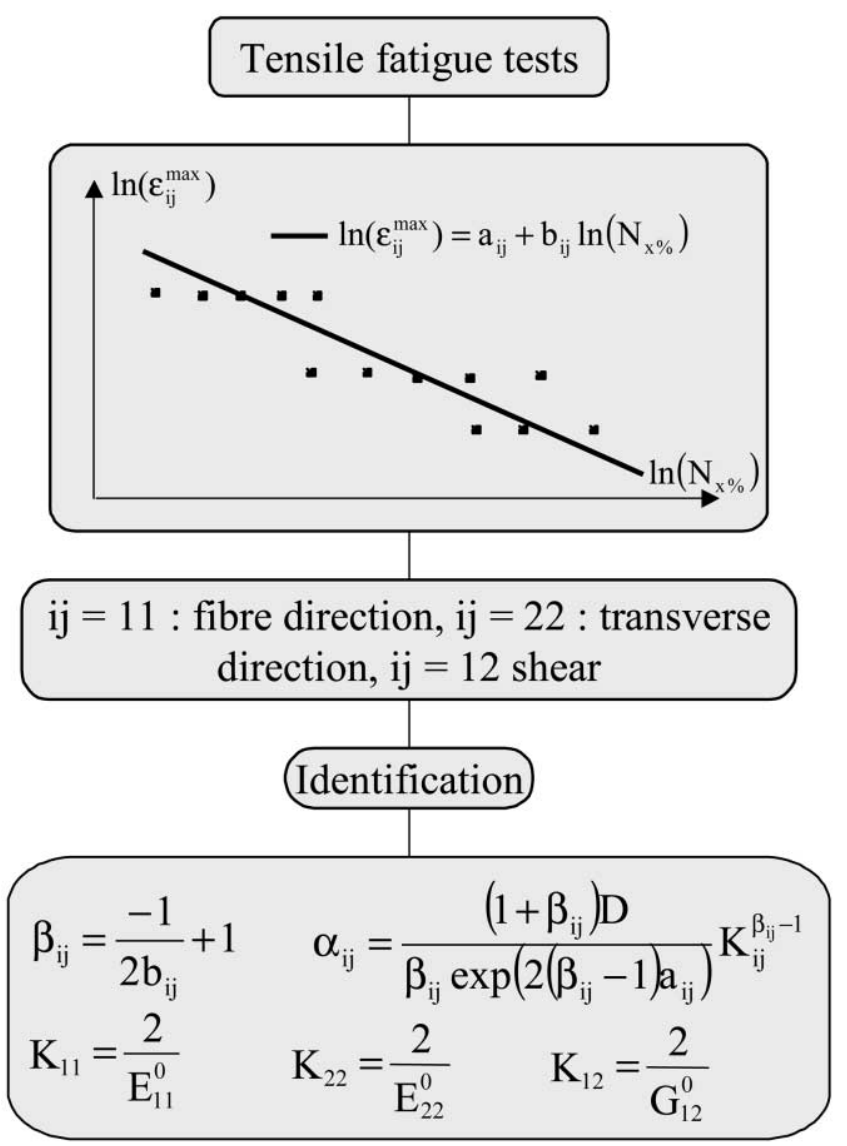

Fig. 5. Method of identification of the parameters $\alpha_{i j}$ and $\beta_{i j}$.

the assembly is made. Only the results of the tensile static and fatigue tests are presented. The focus is on the parameter identification of the damage model.

\subsection{Samples}

The samples used are cut into rectangular plates according to the desired alignment of fibres. The samples with $\left[0^{\circ}\right]$ and $\left[90^{\circ}\right]$ are cut out in plates composed only of plies with $0^{\circ}$ while those with $\left[ \pm 45^{\circ}\right]_{\mathrm{eq}}$ result from laminated plates $[0 / 90]_{\mathrm{eq}}$ cut out with $45^{\circ}$. Dimensions of the samples are shown in Table 1.

In all static tests, strains in the axial and transverse directions were measured. The purpose of this is to:

- determine the evolution of local strains,

- determine Poisson's ratios of materials tested

Table 1

Sample dimensions

\begin{tabular}{llll}
\hline Sample & $\begin{array}{l}L_{\text {useful }} \\
(\mathrm{mm})\end{array}$ & $\begin{array}{l}B \\
(\mathrm{~mm})\end{array}$ & $\begin{array}{l}H \\
(\mathrm{~mm})\end{array}$ \\
\hline $\mathrm{RTM}\left[90^{\circ}\right] \& \mathrm{RTM}\left[ \pm 45^{\circ}\right]_{\mathrm{eq}}$ & 140 & 15 & 5 \\
$\mathrm{RTM}\left[0^{\circ}\right]$ & 150 & 15 & 5 \\
\hline
\end{tabular}




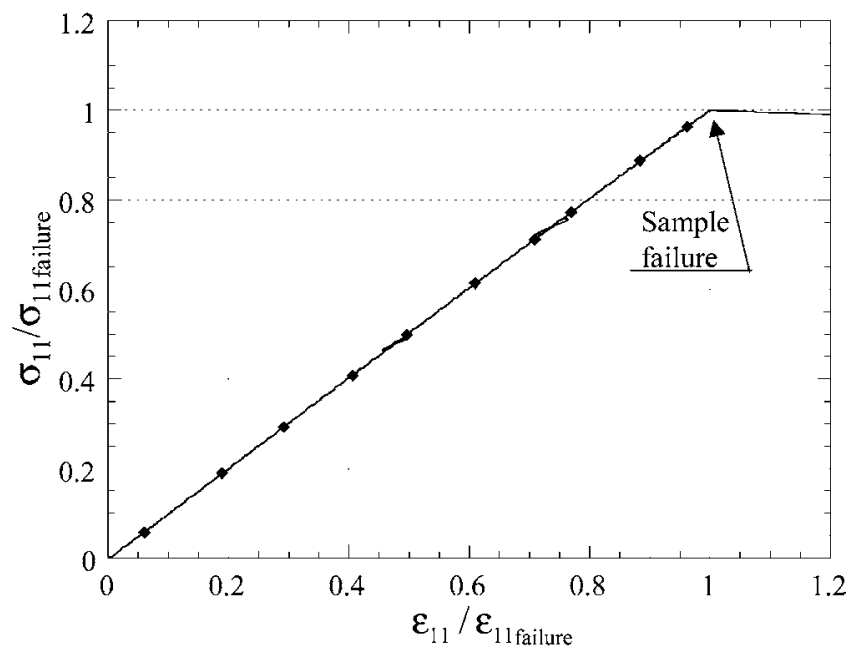

Fig. 6. Static test on $0^{\circ}$.

- verify the symmetry for samples with $\left[0^{\circ}\right]$ or $\left[90^{\circ}\right]$

No instrumentation was used for the fatigue samples. Only information in forces provided by the loading cell is used.

\subsection{Static tests}

A series of static tests was performed in order to determine the static characteristics of the material. The failure strain is also identified. The device test introduced in Section 4.3 is used. Sample displacement is controlled. Induced force is measured. The following behaviour was observed experimentally:

- quasilinear brittle behaviour of the $0^{\circ}$ samples (Fig. 6)

- quasilinear brittle behaviour of the $90^{\circ}$ samples

- non-linear behaviour with the appearance of a plateau to the failure for the $\left[ \pm 45^{\circ}\right]_{\mathrm{eq}}$ samples (Fig. 7)

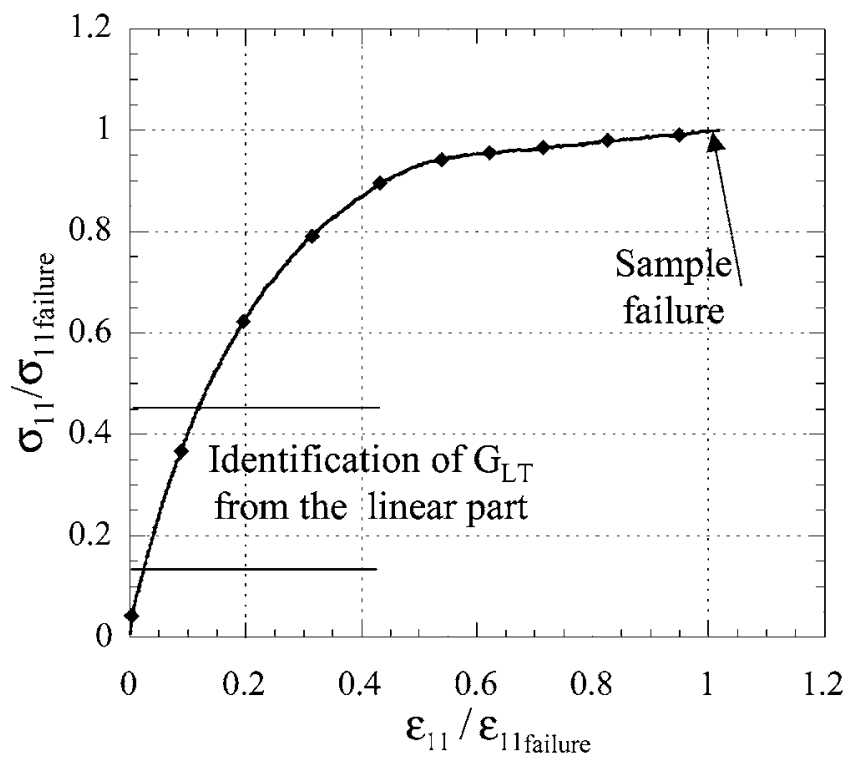

Fig. 7. Static test on $\left[ \pm 45^{\circ}\right]_{\text {eq }}$.
The yield stresses are obtained by the measurement of the induced force. The strains at failure are obtained by the strain gauges. Stress and strain at failure in interlaminar shear are not representative of the tested material because of the plateau (Fig. 7). The appearance of this plateau is due to the stacking of the selected laminate and the reorientation of fibres with $45^{\circ}$ [25]. The identification of moduli in the fibre and transverse directions is carried out easily, while that of the shear modulus must be done on the linear part of the tension diagrams. For shear tests, the loading levels in fatigue are defined so that the applied stress is located below the plateau.

\subsection{Conditions of fatigue tests}

In the case of fatigue tests the following conditions are adopted:

- Displacement ratio: $R=U_{\min } / U_{\max }=0.3$.

- Sinusoidal displacement.

- Variation ranges of the displacement scale ranging between 70 and $20 \%$ of the failure.

- Number of cycles for 5 and $10 \%$ of rigidity loss were measured.

- The loading frequency is equal to $2 \mathrm{~Hz}$ for every loading level.

- All tests are carried out at ambient temperature.

\subsection{Identification of the evolution laws}

From the static results, the loading levels of the fatigue tests were obtained. They verify a loading ratio of 0.3 (Table 2). Three to five samples were tested for each loading level. The number depends on the scattering result. For each test a diagram of "modified Basquin" was carried out at 5 and $10 \%$ of rigidity loss. Figs. 8 and 9 show an example of the diagrams relating to longitudinal and in-shear behaviours.

The fatigue tests represented in a "modified Basquin" diagram allow the identification of the $\alpha_{i j}^{\mathrm{RTM}}$ and $\beta_{i j}^{\mathrm{RTM}}$ parameters. The diagrams corresponding to 5 and $10 \%$ of rigidity loss are represented by the following equation:

$\ln \left(\varepsilon_{i j \max }^{\mathrm{RTM}}\right)=\alpha_{i j}^{\mathrm{RTM}}+b_{i j}^{\mathrm{RTM}} \ln (N x \%)$

where " $x \%$ " is a criterion of examination at 5 or $10 \%$ of rigidity loss.

Fig. 10 represents a rigidity loss for two tests in the transverse direction and with a loading displacement equal to $89 \%$ of failure displacement. This figure shows the extremely brittle character of the composite in this direction. Indeed, the samples in charge with more than $80 \%$ of failure displacement do not undergo any apparent rigidity loss, and break suddenly. Consequently, no number of cycles with $10 \%$ of rigidity loss was noted 
Table 2

Loading levels

\begin{tabular}{lllllll}
\hline Loadingtype & Level & 1 & 2 & 3 & 4 & 5 \\
\hline Longitudinal & $\varepsilon_{\max } / \varepsilon_{\text {failure }}$ & 0.75 & 0.51 & 0.39 & 0.36 & 0.22 \\
Longitudinal & $\varepsilon_{\max } / \varepsilon_{\text {failure }}$ & 0.22 & 0.15 & 0.12 & 0.11 & 0.07 \\
Transverse & $\varepsilon_{\max } / \varepsilon_{\text {failure }}$ & 0.89 & 0.83 & 0.66 & 0.43 & 0.13 \\
Transverse & $\varepsilon_{\max } / \varepsilon_{\text {failure }}$ & 0.26 & 0.25 & 0.2 & 0.4 & 0.12 \\
Shear & $\varepsilon_{\max } \varepsilon_{\text {failure }}$ & 0.65 & 0.55 & 0.5 & 0.15 & \\
Shear & $\varepsilon_{\max } / \varepsilon_{\text {failure }}$ & 0.19 & 0.16 & &
\end{tabular}

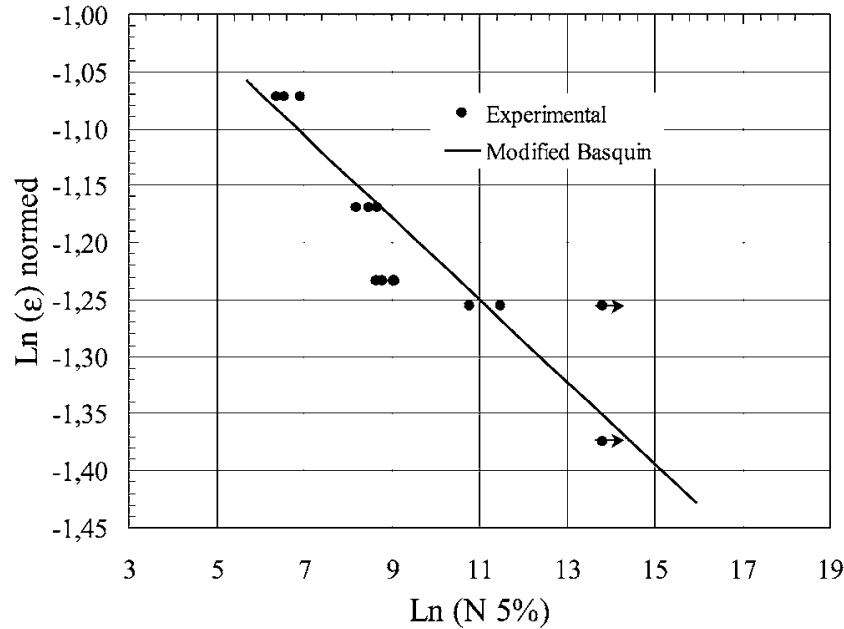

Fig. 8. "Basquin modified" for RTM composite with $0^{\circ}$.

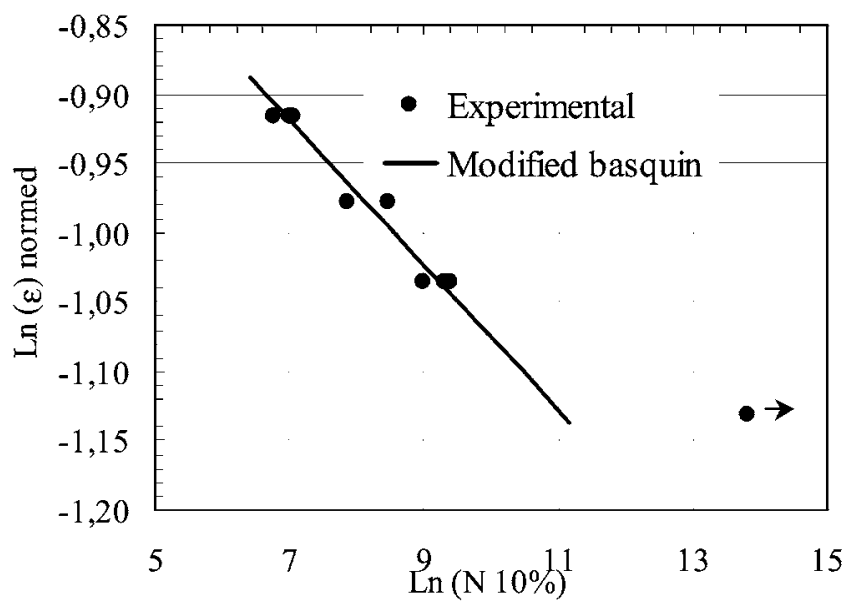

Fig. 9. "Basquin modified" for RTM composite with $\left[ \pm 45^{\circ}\right]_{\mathrm{eq}}$.

for these tests. The levels corresponding to 66 and $43 \%$ (levels 3 and 4, Table 2) of displacement failure correspond to the endurance behaviour, since the samples tested do not undergo any loss of rigidity and resist $10^{6}$ cycles without breaking. For this case of loading the insufficiency of the number of samples tested on the one hand, and levels of loading of fatigue on the other hand, did not allow us to obtain a complete Wöhler diagram. Consequently the coefficients $\alpha_{22}$ and $\beta_{22}$ could not be identified using these tests.

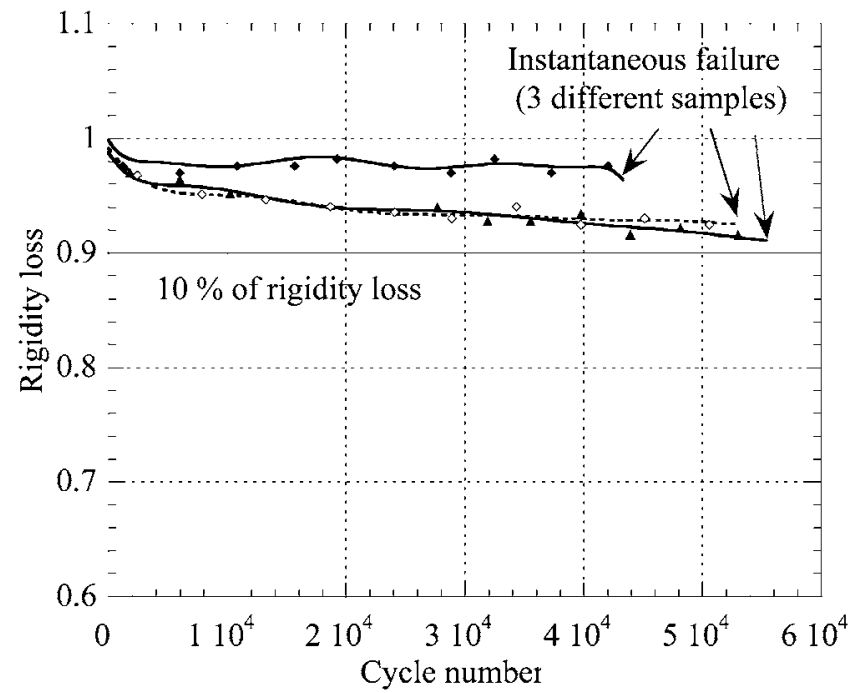

Fig. 10. Brittle character of the matrix behaviour.

Taking into account the problems encountered in these experiments and the brittle character of the matrix, a criterion of the type "all or nothing" will be used for the simulation of behaviour in transverse tension. This criterion arises as follows:

$d_{22}=1$ when $\sigma_{22}=\sigma_{22 \text { failure }}$

$d_{22}=0$ when $\sigma_{22}<\sigma_{22 \text { failure }}$

$d_{22}$ is the damage variable related to the transverse behaviour of the material. $\sigma_{22 \text { failure }}$ is the transverse stress value at failure identified by static tests.

All the results of identification are gathered in Table 3. According to the initial assumptions the coefficients corresponding to shear behaviour in intralaminar and interlaminar are identical.

Table 3

Identification results

\begin{tabular}{llll}
\hline$\alpha_{11}^{\text {RTM }}$ & $1.46 \times 10^{-6}$ & $\beta_{11}^{\text {RTM }}$ & 4.52 \\
$\alpha_{12}^{\text {RTM }}$ & $1.8 \times 10^{-4}$ & $\beta_{12}^{\text {RTM }}$ & 3 \\
$\alpha_{13}^{\text {RTM }}$ & $1.8 \times 10^{-4}$ & $\beta_{13}^{\text {RTM }}$ & 3 \\
$\alpha_{23}^{\text {RTM }}$ & $1.8 \times 10^{-4}$ & $\beta_{23}^{\text {RTM }}$ & 3 \\
$\alpha_{22}^{\text {RTM }}$ & $*$ a & $\beta_{22}^{\text {RTM }}$ & $*$ \\
\hline
\end{tabular}

a Asterisks signify criterion of all or nothing. 


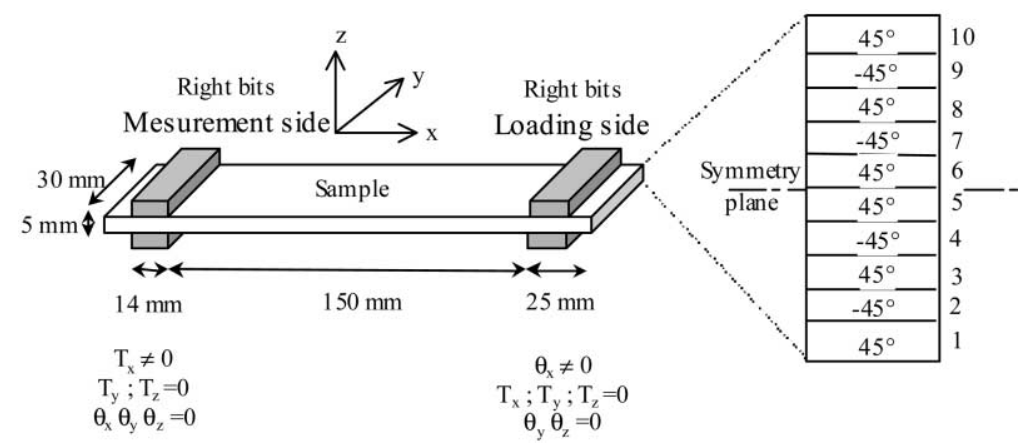

Fig. 11. Diagram of the assembly and degrees of freedom of the system.

\section{Validation of the damage model: fatigue torsion tests on $[ \pm 45]_{S}$}

Once the damage model parameters have been identified, the approach with redistribution of strains can be applied to obtain numerically the structure fatigue behaviour. Numerical results are compared to experimental ones for a $[ \pm 45]_{S}$ composite loaded in fatigue torsion.

\subsection{Experimental tests}

The fatigue behaviour of composite samples loaded in torsion is studied. The composite used is a $5 \mathrm{~mm}$-thick laminate $\left[ \pm 45^{\circ}\right]_{S}$ containing epoxy matrix and unidirectional glass fibres. This composite is worked out by the RTM process. The major interest of these tests resides in the fact that the samples used undergo a complex damage, in particular in interlaminar shear, and the stacking sequence of this laminate's constitutive plies. These tests were carried out at the "Ecole des Mines de St Etienne". The samples are parallelepipedic with nominal dimensions of $200 \times 30 \times 5 \mathrm{~mm}^{3}$. The testing machine is equipped with a system of prismatic grips having a contact surface of $30 \times 14 \mathrm{~mm}^{2}$ with the samples. Their useful area is $150 \mathrm{~mm}$ long. Fig. 11 represents the diagram of the assembly used and the dimensions of the sample. These tests were controlled in imposed angular displacement.

In order to determine the levels of loading imposed in fatigue, a series of tests into quasi-static was carried out. The rupture of the samples is characterised by cracks occurring in the centre of the small edge of the cross-section. Those happen in the areas which are rich of resin and then occur towards the core of the sample (Fig. 12).
Fatigue tests are carried out under the following experimental conditions:

- Frequency: $1 \mathrm{~Hz}$

- Loading ratio: $R=\theta_{\min } / \theta_{\max }=0.3 \quad(\theta$ being the imposed angle)

- Temperature: room temperature

- Servo-control: angular undulated displacement

- Maximum number of cycles: 1 million.

The samples are tested on 5 levels of initial maximal couples. Fig. 14 gathers all test results in a Wöhler diagram. The points being reproduced in the latter result from an examination with 5 and $10 \%$ of rigidity loss. We notice the small scattering of all the results. The first level of loading can be compared to low cycle fatigue and the last level to that of high cycle fatigue. The three intermediate levels can be represented by a quasi linear distribution in a logarithmic scale, which leads to identification of the fatigue domain.

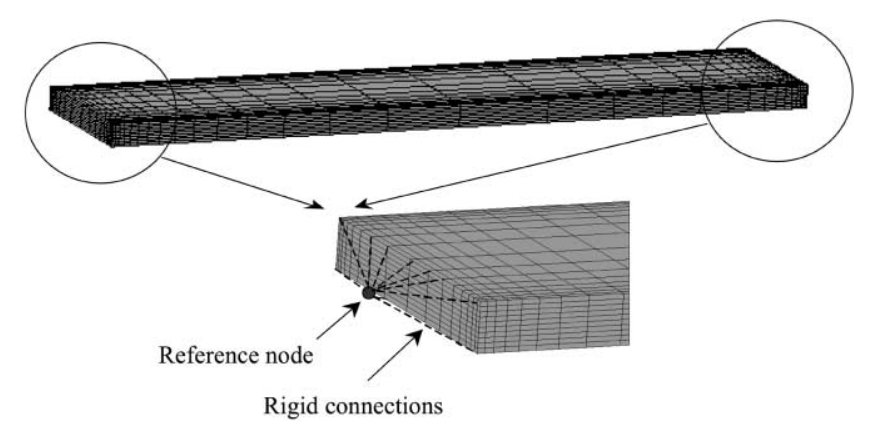

Fig. 13. FE mesh and boundary conditions.

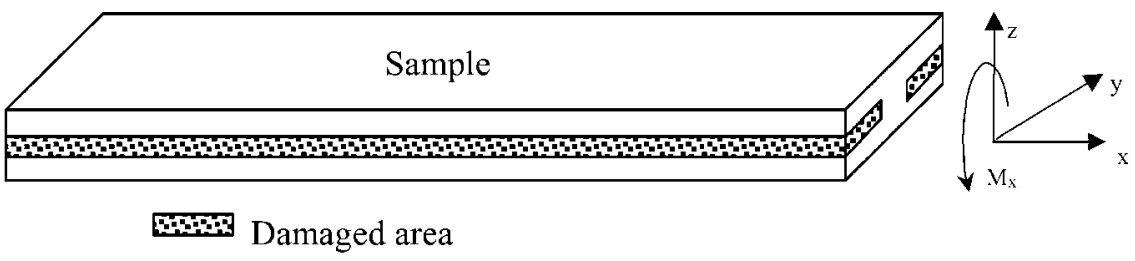

Fig. 12. Damage of the samples in static and fatigue cases. 


\subsection{Numerical test}

\subsubsection{FE model}

The dimensions of the modelled samples are $150 \times 30 \times 5 \mathrm{~mm}^{3}$. The software Abaqus is used with C3D8 elements (bricks with 8 nodes with linear interpolation). The mesh is refined near the wedges and the free edges (Fig. 13). This model comprises 17,500 d.o.f. and 4800 elements. The loading is applied to reference nodes, located symmetrically, in the right and left sides. Rigid relationships were defined between the reference node and the superior and inferior edges of the sample extremity (Fig. 13).

\subsubsection{Simulation of the fatigue behaviour}

The damage model parameters used are collated in Table 3 (coefficients for RTM material). A test with imposed angular displacement is simulated with values defined in Section 5.1. This imposed numerical loading corresponds to the maximum level of the experimental loading. Fig. 14 represents a comparison between the experimental and numerical Wöhler curves. Two main tendencies can be released from this representation:

- The identified coefficients $\alpha_{i j}$ and $\beta_{i j}$ are only valid for the fatigue domain. Indeed, the simulation overestimates the low cycle fatigue domain and conversely endurance domain. In fact, the model is based on the coefficients $a_{i j}$ and $b_{i j}$ which were determined over the high cycle fatigue regime.

- The case of torsion in fatigue highlights the need to take into account the load transfer phenomena while modelling.

\section{Conclusions}

A damage model based on generalised standard material thermodynamics was used. A potential dissipation based on Norton formulation was implemented. It takes into account various damage modes and parameters which are related to the material behaviour and loading conditions, such as the stress ratio and loading frequency. The damage model associated with structural analyses and the introduction of elementary mesoscopic scale modelling make it possible to solve the problems involved in the gradients of strains and stresses through laminate plies.

The characterisation and identification of the presented damage model were carried out. Tensile tests were defined for the fatigue behaviour identification in the fibre direction. The sensitivity of these tests to microdefects was highlighted. It appears that the manufacturing process and the cutting of samples should be controlled during the characterisation.

This study showed the interest of modelling with updating of the strain field, as well as the capacity of taking into account the load redistribution phenomena during the fatigue behaviour simulation. In the case of torsion tests on $[ \pm 45]_{S}$ laminates, the Wöhler diagram resulting from the iterative approach shows a good coherence with the experimental results in the fatigue domain. It should be stressed that the suggested model does not integrate the low cycle fatigue behaviour. Taking account the latter would require:

- Additional identification tests

- Norton's law enrichment

- Load level consideration.

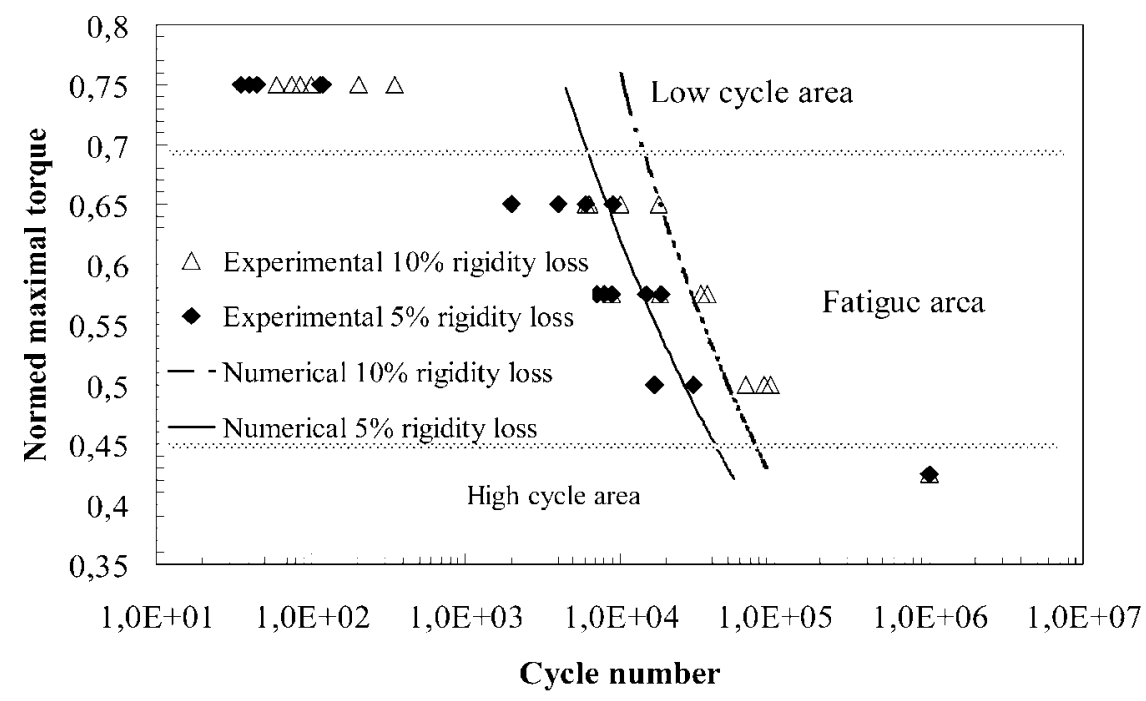

Fig. 14. Comparison of the numerical and experimental results. 


\section{Acknowledgements}

This work was financed by Renault and monitored by P. Lory and V. Bobet (Renault). The authors gratefully acknowledge this support.

\section{References}

[1] Dang Van. Sur la résistance à la fatigue des métaux. Extrait de sciences et techniques de l'armement. Mémorial de l'artillerie. Troisième fascicule; 1973.

[2] Dang Van et al. Critère d'amorçage en fatigue à grand nombre de cycles sous sollicitations multiaxiales. Journées de commission fatigue de la société de métallurgie. Rapport RE 1123. Paris; May 1984.

[3] Asundi A, Chow CL. Analysis of damage field and actual stressstrain field in composite plate by moire interferometry. In: ICCM-7., 1991:668-703.

[4] Shokrieh MM, Lessard LB. Multiaxial fatigue behaviour of unidirectional plies based on uniaxial fatigue experiments. Part I and II. Int J Fatigue 1997;19:201-17.

[5] Haplin JC et al. Analysis of the test methods for high modules fibers and composites. In: ASTM STP 521., 1997:5-64.

[6] Whitney JM. Fatigue of fibrous composite materials. In: ASTM STP 723., 1981:133-51.

[7] Reifsnider KL. In: Fatigue of composite materials., 1991:11-77.

[8] Hashin Z. Fatigue failure criteria for unidirectional fiber composites. Trans ASME 1981;48(December).

[9] Lemaitre J, Chaboche JL. Mécanique des matériaux solides. Edition Dunod, 1985.

[10] Ladeveze P. Sur la mécanique de l'endommagement des composites. Compte Rendu des JNC 1986;5.

[11] Thionnet A. Endommagement dans les matériaux composites stratifiés. Thesis, Paris VI; 1991.
[12] Wang SJ. Etude de l'endommagement d'un composite unidirectionnel verre/epoxy. Thesis, ECL; 1990.

[13] El Kadi H, Ellyin F. Effect of stress ratio on the fatigue of unidirectional glass fiber/epoxy composite laminae. Composites 1994;25(10).

[14] Nakada M, Miyano Y. Influence of stress ratio on fatigue behaviour in the transverse direction of unidirectional CFRPS. J Composite Mater 1995;29(14).

[15] Lalanne C. Dommage par fatigue. In: Hermes, 1999.

[16] Ladeveze $P$ et al. Une nouvelle approche des composites par la mécanique de l'endommagement. ENS Cachan, 1993.

[17] Linhonne C. Un modèle de comportement élastoplastique endommageable pour les structures composites stratifiés. La Recherche Aérospatiale 1996;2.

[18] Guilmineau P et al. Un modèle d'endommagement des matériaux composites sous sollicitions quasi-statiques et cycliques basé sur la description physique du défaut. In: JNC-8., 1992.

[19] Devries F. Une approche micro-macro de l'endommagement de fatigue des composites unidirectionnels. In: Fatigue des matériaux composites à matrice organique. AMAC, 1990:64-72.

[20] Masmoudi W. Mutation de produit: Conception et modélisation d'un bogie de TGV en matériaux composites. Thesis, ENSAM; 1993.

[21] Hahn HT, Erikson J. Characterization of composite laminates using tubular specimens. In: Report AFML-TR-77-144. OH, USA: Air Force Material Laboratory, Wright Patterson Air Force Base, 1977.

[22] Lee S, Munro M. Evaluation of in-plane shear test method for composite materials by decision analysis technics. Composites 1990;17:13-22.

[23] Boehler JP, El Aoufi L. Problèmes expérimentaux pour la caractérisation mécanique des matériaux composites. In: Caractérisation mécanique des composites: Extensométrie appliquée aux composites. Edition Pluralis, 1989:3-16.

[24] Sedrakian A. Contribution à la modélisation du comportement en fatigue des pièces composites stratifiés. Thesis, ENSAM; 2000.

[25] Wisnom MR. The effect of fibre rotation in $\pm 45^{\circ}$ tension tests on measured shear properties. Composites 1995;26(1):25-32. 